\title{
ÉCRIRE, ÉCRIRE, POUROUOI?
}

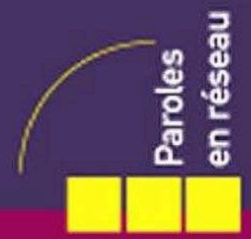

Entretien avec

Amélie Nothomb

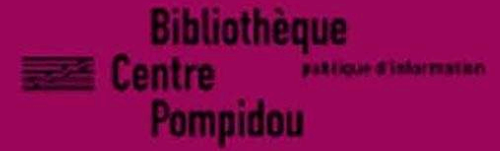




\section{Écrire, écrire, pourquoi ? Amélie Nothomb}

Entretien avec Josyane Savigneau

\section{Amélie Nothomb et Josyane Savigneau}

DOI : 10.4000/books.bibpompidou. 1173

Éditeur : Éditions de la Bibliothèque publique d'information Année d'édition : 2010

Date de mise en ligne : 17 janvier 2014

Collection : Paroles en réseau

ISBN électronique : 9782842461812

\section{Donestition}

http://books.openedition.org

Édition imprimée

ISBN : 9782842461317

Nombre de pages : 24

\section{Référence électronique}

NOTHOMB, Amélie ; SAVIGNEAU, Josyane. Écrire, écrire, pourquoi ? Amélie Nothomb : Entretien avec Josyane Savigneau. Nouvelle édition [en ligne]. Paris : Éditions de la Bibliothèque publique d'information, 2010 (généré le 02 février 2021). Disponible sur Internet : <http://books.openedition.org/ bibpompidou/1173>. ISBN : 9782842461812 . DOI : https://doi.org/10.4000/books.bibpompidou. 1173.

(c) Éditions de la Bibliothèque publique d'information, 2010

Conditions d'utilisation :

http://www.openedition.org/6540 


\title{
Écrire, écrire, pourquoi?
}

\author{
Cycle de rencontres organisé par la Bpi \\ Lundi 26 janvier 2009 \\ Invitée: Amélie Nothomb \\ Entretien avec Josyane Savigneau
}

Bibliothèque

Centre

Pompidou

\section{8}

Liberté $\cdot$ Egalité $\cdot$ Fraternité RÉPUBLIQUe FrAnÇAISE

publique d'information

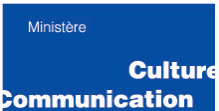




$\begin{array}{ll}\begin{array}{l}\text { Président } \\ \text { du Centre Pompidou } \\ \text { Alain Seban }\end{array} & \begin{array}{l}\text { Colloque } \\ \text { Conception } \\ \text { et organisation } \\ \text { Francine Figuière }\end{array} \\ \begin{array}{l}\text { Directrice générale } \\ \text { du Centre Pompidou }\end{array} & \begin{array}{l}\text { Publication } \\ \text { Chargée d'édition } \\ \text { et mise en page }\end{array} \\ \begin{array}{l}\text { Directeur de la Bpi } \\ \text { Patrick Bazin }\end{array} & \text { Sandra Bocquier }\end{array}$

Responsable du pôle

Action culturelle

et Communication

Philippe Charrier

Chef du service

Animation

Emmanuelle Payen

Chef du service

Édition/Diffusion

Arielle Rousselle

Avertissement :

L'adaptation de cet entretien de l'oral à l'écrit a pu entraîner des modifications de style ou de forme, ce qui explique les différences éventuelles entre cette publication et l'enregistrement réalisé lors de la rencontre.

Écoutez les rencontres sur le site:

http://archives-sonores.bpi.fr.

Catalogue des éditions:

http://editionsdelabibliotheque.bpi.fr/

Distribution numérique

par GiantChair.com

(c) Éditions de la Bibliothèque

publique d'information/Centre

Pompidou, 2010.

ISBN 978-2-84246-131-7

ISSN 1765-2782 


\title{
Amélie Nothomb
}

\author{
Entretien avec Josyane Savigneau \\ Journaliste au Monde
}

Amélie Nothomb est née en 1967 à Kobé au Japon. Sa famille fait partie de l'aristocratie belge. Fille d'ambassadeur, son enfance est rythmée par d'incessants déménagements au gré des affectations paternelles, de la Chine à la Birmanie, en passant par New York. Elle racontera une partie de son enfance dans ses romans Métaphysique des tubes, Le Sabotage amoureux et Biographie de la faim. Elle est profondément marquée par le Japon où elle a passé les cinq premières années de sa vie et où elle retournera après ses études pour travailler comme interprète, période qu'elle transposera dans ses romans Stupeur et Tremblements et Ni d'Éve ni d'Adam.

Ce n'est qu'à l'âge de dix-sept ans qu'elle découvre la Belgique. Elle y fait des études de philologie romane à l'Université libre de Bruxelles. En 1992, elle publie chez Albin Michel son premier roman, Hygiène de l'assassin, qui connaît un énorme succès et sera adapté au cinéma. Se définissant elle-même comme une " graphomane malade de l'écriture ", elle dit écrire trois romans par an pour n'en faire paraître qu'un, soit dix-sept romans à ce jour, autobiographiques ou purement fictionnels. Son œuvre, traduite en quarante langues, est caractérisée par une profondeur alerte, un style romanesque et décalé à l'humour subtil qui place son lecteur directement face à ses pulsions intérieures.

Aux éditions Albin Michel

puis au Livre de poche (LGF)

Hygiène de l'assassin, 1992, prix René Fallet, prix Alain-Fournier

Le Sabotage amoureux, 1993, prix de la Vocation, prix Chardonne

Les Combustibles, théâtre, 1994

Les Catilinaires, 1995

Péplum, 1996

Attentat, 1997

Mercure, 1998

Stupeur et Tremblements, 1999, Grand Prix du roman

de l'Académie française

Métaphysique des tubes, 2000

Cosmétique de l'ennemi, 2001

Robert des noms propres, 2002

Antéchrista, 2003

Biographie de la faim, 2004

Acide sulfurique, 2005

Journal d'Hirondelle, 2006

Ni d'Ève ni d'Adam, 2007, prix de Flore

Le Fait du prince, 2008, Grand Prix Jean Giono

Le Voyage d'hiver, Albin Michel, 2009

Une forme de vie, Albin Michel, 2010

Amélie Nothomb parle de son dernier roman, Une forme de vie, sur: http://www.albin-michel.fr/rentree-litteraire/auteurs/amelie-nothomb/ 
Josyane Savigneau : Lorsqu'on a demandé un jour à Samuel Beckett pourquoi il écrivait, il a répondu: «Bon qu’à ça. » Que répondriez-vous à cette question?

Amélie Nothomb : Je ne voudrais pas le plagier mais j'ai bien peur qu'il ne faille dire la même chose. J'ai essayé de faire autre chose. Preuve en est dans un de mes livres, Stupeur et Tremblements, je tente d'être une employée de bureau japonaise modèle. Ce fut un véritable désastre et il a bien fallu que je me reconvertisse. Je ne sais pas si je suis bonne en écriture mais une chose est sûre: en dehors de l'écriture, je ne sais strictement rien faire.

Josyane Savigneau : Dans Biographie de la faim, vous dites que quand vous êtes arrivée en Belgique, à dix-sept ans, c'est le pays que vous avez le moins compris et que cela vous a motivé pour écrire.

Amélie Nothomb : Il est vrai que d'arriver dans un pays, censé être le mien, dans lequel je n'avais jamais mis les pieds, m’a motivée à écrire. Ce pays, censé contenir mon identité, la contenait encore moins que les autres pays. J'ai fini par comprendre que j’y avais une identité. Mais pour découvrir ce mystère absolu, j'ai écrit. Quand on ne comprend rien, on écrit pour faire une espèce de diagnostic de la situation. Plus mystérieux, peut-être, est le premier roman, écrit à mon arrivée en Belgique, qui racontait l'histoire d'une omelette spatiale. Je ne sais pas si c'était un diagnostic sur la situation belge ou mondiale.

Josyane Savigneau : Ce roman n'a pas été publié. Vous n'avez commencé à Entretien avec publier qu'à vingt-cinq ans et chaque année depuis. Cependant, vous avez Josyane Savigneau écrit avant et composez plus d'un livre par an.

Amélie Nothomb : Oui, et heureusement. Si mes calculs sont exacts, j’ai publié dix-sept livres, ce qui est beaucoup. Or, je suis en train d'écrire mon soixante-cinquième livre. Imaginez donc l'obscénité, si à quarante et un ans, je n'avais pas publié dix-sept livres sur mes soixante-cinq: ce serait monstrueux! Heureusement, je n'ai pas commis cette erreur. Mais je ne fais pas partie des écrivains qui se croient obligés de publier tout ce qu'ils écrivent. Je dirais même que, chez moi, le désir de publications est plutôt un accident de parcours par rapport à l'acte d'écriture. J'ai l'intime conviction que le patrimoine mondial de la littérature se passe fort bien des quarante-huit manquants. J'ai déjà pris des mesures testamentaires pour que les livres qui ne sont pas publiés à ce jour ne le soient pas, même en cas de décès. Je n'ai pas le désir de les publier. En outre, je me fie beaucoup aux cambrioleurs. J'ai été cambriolée deux fois à Bruxelles et mes manuscrits non publiés, qui traînent dans des caisses en carton par terre, n’ont pas été volés.

Josyane Savigneau: Comme Jacques Lacan ${ }^{1}$, diriez-vous « poubellication " plutôt que publication? 
Amélie Nothomb : J'ai appris ce mot fascinant de "poubellication » par un psychanalyste qui a écrit une analyse lacanienne de mon œuvre ${ }^{2}$. J'adore cette idée car les poubelles sont un peu notre archéologie.

Josyane Savigneau: Hygiène de l'assassin, votre premier livre publié, a été un succès immédiat. Avez-vous été étonnée?

Amélie Nothomb : Complètement! Déjà, que je trouve un éditeur, somme toute aussi facilement, n'allait pas de soi; en plus, que ce livre connaisse d'emblée le succès qu'il a eu et qu'il continue d'avoir... J'imaginais que ce livre était extrêmement élitiste. Je me trompais puisqu’il s'est avéré qu’il ne l'était pas et j'étais heureuse d'être un écrivain grand public.

Josyane Savigneau: Justement, écrire pour un public large a-t-il changé quelque chose pour vous?

Amélie Nothomb: Non, au moment où j'écris je ne sais jamais si ce sera destiné à la publication ou aux caisses en carton de l'appartement de Bruxelles.

Josyane Savigneau : Comment faites-vous ce choix?

Amélie Nothomb : Généralement aux mois de janvier-février, je relis ce que j'ai écrit dans l'année. À ce moment-là, je me pose la question de savoir si j'ai envie de publier l'un des trois ou quatre manuscrits écrits dans l'année.

Amélie Nothomb

Entretien avec

Josyane Savigneau

Josyane Savigneau: Dès Hygiène de l'assassin, on peut dire que vous avez
réglé cette question du succès. Un vieil écrivain répond sur cette question: " Je n'explique pas les raisons de mon succès. Les usines d'armement vendent chaque jour des milliers de missiles à travers le monde, et cela ne les fait pas réfléchir non plus. » Est-ce votre réponse?

Amélie Nothomb : C'est une réponse très mégalomane d'imaginer que dans mes livres il y aurait un pouvoir de nuisance égale à celui des usines d'armement. Cependant, il est vrai qu'Hygiène de l'assassin est un livre que j'ai entièrement écrit à partir du postulat que les gens ne lisaient pas. Depuis que ce livre est paru, j'ai tous les jours la preuve que mon postulat était faux.

Josyane Savigneau : Vous avez parlé de votre premier manuscrit, L'Omelette spatiale, qui semble être de la science-fiction. Vous y êtes revenu avec Péplum. Allez-vous en écrire d'autres?

Amélie Nothomb : La science-fiction est le contraire de tout le reste de la littérature, c'est-à-dire des aventures extraordinaires qui arrivent à des gens ordinaires. Cependant, je suis incapable de savoir si j'en écrirai d'autre, mais c'est possible. 
Josyane Savigneau: Écrivez-vous à la main?

Amélie Nothomb : J'écris à la main effectivement. Je ne possède pas d'ordinateur et maintenant que j'ai de bonnes relations avec mon éditeur, c'està-dire qu'il n'est pas en état de me refuser quelque chose, je lui apporte le manuscrit à l'état de manuscrit, puis il est dactylographié.

Josyane Savigneau: Je pense que vous avez raison et que l'ordinateur est déjà une écriture collective. C'est déjà être dans la position du récepteur. Il n'y a plus de rapport physique à l'écrit, comme celui que vous avez.

Amélie Nothomb: Je partage tout à fait cet avis sur la notion de rapport physique à l'écrit. Cependant, votre réflexion va beaucoup plus loin que la mienne. La vérité est que face à un ordinateur, je suis même incapable de l'allumer.

Josyane Savigneau : Votre dernier livre, Le Fait du prince, est une comédie délicieuse sur l'usurpation d'identité. En fait, c'est un jeu sur l'absurde et les quiproquos. J'aime beaucoup la phrase que vous avez choisie pour la quatrième de couverture: « Il y a un instant entre la quinzième et la seizième gorgée de champagne où tout homme est un aristocrate. » Dans ce livre, il y a beaucoup de variations sur le champagne: une femme dont la nourriture principale est le champagne; les différentes marques de champagne, etc.

Amélie Nothomb: Le Fait du prince est beaucoup de choses à la fois mais 6 c'est un peu ma version champagnisée de Vingt mille lieues sous les mers de Amélie Nothomb Jules Verne. On peut considérer que je suis un peu dans le bathyscaphe; je Entretien avec suis le capitaine Nemo qui explore la piscine de champagne, avec beaucoup Josyane Savigneau de science.

Josyane Savigneau : Avez-vous réussi à créer un imposteur complètement exponentiel?

Amélie Nothomb: Complètement. Je parle de ma vie et du fait que tous les écrivains ont le sentiment d'être des imposteurs. À plus forte raison quand le livre rencontre le succès. Une certaine mauvaise conscience fait écrire ce genre de livre.

Josyane Savigneau: Dans ce livre justement, il y a beaucoup de dialogues loufoques. De manière générale, il semblerait que vous aimiez les dialogues.

Amélie Nothomb: Je trouve fascinant d'explorer des personnages à travers ce qu'ils ont à se dire et non forcément par leurs monologues intérieurs. Placer deux personnages ensemble, ne pas les décrire physiquement et explorer leurs échanges. En général, c'est étrange. Dans la vie de tous les jours, les gens se disent des choses très curieuses. J'écoute souvent les gens, surtout dans le métro: les conversations sont vraiment incroyables.

Josyane Savigneau : Vous en servez-vous directement? 
Amélie Nothomb: Non, mais cela verse de l'eau à mon moulin. Je vois bien que les choses que j'écris ne sont pas beaucoup plus hallucinantes que ce que les gens se racontent en vrai.

Josyane Savigneau : Il est vrai que parfois des situations réelles paraissent extraordinaires et seraient perçues comme exagérées dans un roman. Par moments, justement dans certains de vos dialogues, n'y a-t-il pas un effet de ce réel étrange?

Amélie Nothomb : J'ai la folle prétention de croire que mes livres parlent de la réalité.

Josyane Savigneau: Il y a une phrase d'un des personnages, dans Le Fait du prince: "Il faut glisser des fictions dans la vie comme des enfants. " Le faites-vous?

Amélie Nothomb : Je le fais tout le temps. Les fictions peuvent être parfaitement anodines et peuvent donner lieu à des conséquences incroyables. Je considère comme une fiction glissée dans la réalité le fait d'être interviewée par Josyane Savigneau.

Josyane Savigneau : La Métaphysique des tubes est une histoire très réaliste, où vous êtes Dieu et avez décidé de ne rejoindre le commun des mortels qu'au bout d'un certain temps.

Amélie Nothomb: Je pense que c'est une histoire commune. La seule différence entre les gens et moi est que je m'en souviens. J'ai le souvenir Amélie Nothom assez précis d'avoir été Dieu pendant les deux premières années et demie de Josyane Savigneau ma vie. Je suis persuadée que nous sommes tous passés par là: bébés, nous étions tous Dieu; et à un moment, pour des raisons qui paraissent toujours accidentelles, il a fallu y renoncer.

Josyane Savigneau : Pour vous, est-ce le chocolat apporté par votre grandmère qui vous a obligé à vous rallier à l'humanité ordinaire?

Amélie Nothomb : Non. Je pense que le chocolat m'a permis de me rallier à cette humanité ordinaire avec le sourire. Je pense que j'avais déjà compris que ce n'était peut-être pas moi, Dieu. Seulement, cette idée m’était insupportable, d'où les hurlements que je poussais sans trêve dans mon berceau. Ce chocolat belge m'a fait réaliser que je n'étais pas Dieu, mais ce n'était pas si grave puisqu'il existait le plaisir.

Josyane Savigneau : Ce chocolat fut également un déclencheur de mémoire puisqu'à partir de ce moment-là, ne dites-vous pas vous souvenir de tout ce qui est arrivé?

Amélie Nothomb: Je pense que le plaisir est puissamment mnémotechnique. C'est aussi pour ça que pour entretenir ma mémoire, je m'octroie de grandes doses de plaisir quotidien, uniquement dans un but mnémotechnique bien sûr. 
Josyane Savigneau : En avez-vous parlé par la suite avec votre grand-mère, de cette affaire de chocolat?

Amélie Nothomb: Je n'ai pas eu le temps de lui en parler puisque, malheureusement, elle est morte trois mois plus tard. Cependant, j'ai l'impression qu'elle a su qu'elle était très importante dans ma vie.

Josyane Savigneau: Ne retrouve-t-on pas le chocolat dans Biographie de la faim?

Amélie Nothomb: Il y a deux ingrédients que l'on retrouve de plus en plus dans mes livres: le chocolat et le champagne. C'est normal puisqu'ils constituent la base de mon alimentation. Comme dans les natures mortes ou les grottes de Lascaux, on dessine en quelque sorte ce que l'on mange. Le chocolat, l'alimentation des dieux, joue un rôle capital dans ce que j'écris. Je crois qu'il entretient chez moi la nostalgie divine.

Josyane Savigneau : Biographie de la faim paraît être un livre extrêmement important pour plusieurs raisons. L'une d'elles n'est-elle pas l'effroi éprouvé à sortir de l'enfance, que l'on voit dans beaucoup de vos livres et dans celuici particulièrement?

Lecture de Biographie de la faim par Amélie Nothomb:

Jamais était le pays que j'habitais. C'était un pays sans retour. Je ne l'aimais pas. Le Japon était mon pays, celui que j'avais choisi, mais 8 lui ne m’avait pas élue...

(Écouter la suite sur archives sonores, repère: 17 min 11 s)

Amélie Nothomb

Entretien avec

Josyane Savigneau

Josyane Savigneau: Dans ce livre, on retrouve le chocolat et Dieu. Ne suffitil pas d'avoir en bouche du très bon chocolat, non seulement pour croire en Dieu mais aussi pour se sentir en sa présence? «Dieu n'est pas le chocolat mais la rencontre entre le chocolat et le palais capable de l'apprécier.» Cela devient déjà plus complexe.

Amélie Nothomb: C'est de la métaphysique de haut niveau. Toutes ces phrases ont été reprises par des chocolatiers belges. Ils me veulent tous comme emblème, ce que j'accepte car je suis payée en chocolat.

Josyane Savigneau: Dans Biographie de la faim, il y a également de nombreux portraits de gens que vous avez aimés, celui de votre sœur notamment. Il $\mathrm{y}$ a cette scène très drôle où votre frère est envoyé en Belgique tandis que vous, les filles, allez partir à New York. Votre sœur et vous le regardez partir avec vos parents qui le conduisent à l'aéroport. Votre sœur dit: « Tu te rends compte, le pauvre, il va dans une prison belge alors que nous, nous allons vivre à New York. » Sur ce, vous répondez: «Il y a une justice. " S'ensuit le portrait de Juliette:

Juliette, dix ans et demi, était mon rêve. Quand on lui demandait ce qu’elle voulait devenir à l'âge adulte, elle répondait : « Fée ». En 
vérité, elle en était une de toute éternité, comme le prouvait son joli visage toujours dans la lune...

(Écouter la suite sur archives sonores, repère: 20 min 36 s)

Après ce passage, vous faites une description magnifique de New York, ville qui vous a exaltée. Est-ce toujours une ville que vous aimez?

Amélie Nothomb : Oui, j'éprouve toujours un bonheur intense à l'idée d'y retourner, notamment pour la parution en américain de Ni d'Éve ni d'Adam, sous le titre de Tokyo fiancée. Ce sera un plaisir d'aller défendre ce livre au titre incongru dans une ville si parfaitement géniale.

Josyane Savigneau : N'est-ce pas à New York que vous tentez de partir à la conquête de votre gouvernante, Inge? Quel âge aviez-vous?

Amélie Nothomb : Je suis arrivée à New York à l'âge de huit ans. Cependant, mon premier coup de cœur était en Chine: Elena, qui allait dans la même école que moi, dont je parle dans le Sabotage amoureux. Mes parents avaient pris pour habitude d'engager des gouvernantes pour ma sœur et moi. Ils sortaient beaucoup, il fallait donc que quelqu'un s'occupe de nous. À New York, non seulement le frère est envoyé dans une prison belge, premier bonheur, et la gouvernante est divine.

Josyane Savigneau : Dans Biographie de la faim, il y a beaucoup d'humour mais aussi du tragique. Par exemple, vous dites: "La mort contenue dans 9 la vie m'effraya. " Est-ce toujours le cas?

\section{Amélie Nothomb}

Entretien avec

Amélie Nothomb: On comprend vite quand on est petit que la vie est Josyane Savigneau " pleine de mort » et c'est effrayant. La lecture de Nietzsche aide à digérer cette grande découverte mais, même en acceptant la théorie de «l'éternel retour », au quotidien être conscient de la mort reste très dur.

Josyane Savigneau: Puis vous devez quitter New York. Vous allez en Birmanie et dites:

J'eus treize ans en Birmanie. [...] Mon corps se déforma. Je grandis de douze centimètres en un an. Il me vint des seins, grotesques de petitesse, mais c'était déjà trop pour moi...

(Écouter la suite sur archives sonores, repère: $24 \mathrm{~min} 12 \mathrm{~s}$ )

Amélie Nothomb : Treize ans est un âge abominable et cette expérience est moins singulière qu'on ne le pense. Je pense que la littérature a beaucoup menti à ce sujet, particulièrement pour l'adolescence des filles, mais pas uniquement. Dans la littérature lue à l'époque, on voyait des petites filles folles de joie de devenir des jeunes filles. Je ne l'ai pas du tout vécu ainsi. Maintenant que je suis adulte et que j'en parle beaucoup avec des adultes, ils me disent la même chose. Ils l'ont vécu de manière complètement traumatisante. C'est peut-être l'un des ingrédients pour lesquels j'ai beaucoup de lecteurs: ils se sont reconnus dans cette vision apocalyptique du passage à l'âge adulte. 
Josyane Savigneau: Cette sensation de désastre a-t-elle motivé votre décision de ne plus manger, le jour de la sainte Amélie, le 5 janvier 1981?

Amélie Nothomb: C'était certainement lié, mais renforcé par le fait que cette expérience était vécue avec ma sœur. Il y avait donc aussi une valeur d'acte amoureux, car un acte commis ensemble.

Josyane Savigneau : Il y avait aussi une volonté de rassemblement et de totalisation, puisque vous dites: "Cette déperdition de soi s'accompagnerait d'une rétention. La Loi stipulait aussi qu'à dater de ce jour, je n'oublierais plus aucune émotion de ma vie. »

Amélie Nothomb : À treize ans, on prend des résolutions titanesques. J'en avais donc pris le pari insensé. Ce n'est pas loin d'avoir fonctionné. En effet, j'ai tendance à garder les émotions de ma vie de manière très forte.

Josyane Savigneau : Chez vous, n'était-ce pas un refus de sortir de l'enfance et de la femme que vous étiez en train de devenir, plutôt que l'idée d'une femme parfaite qui serait dans la maigreur? Étiez-vous gagnée par cette modélisation de la "belle fille "?

Amélie Nothomb: Loin de moi l'idée d'une "femme parfaite ». Je n'ai jamais eu beaucoup d'ambition dans ce domaine.

Josyane Savigneau: N'aviez-vous pas décidé de "manger les mots " par 10 substitution?

Amélie Nothomb : Oui. J'ai entièrement lu le dictionnaire à cet âge. Il est Josyane Savigneau vrai que les mots nourrissent.

Josyane Savigneau: Sur cette idée de la rétention, du renoncement, vous dites: «L'ascèse n'enrichit pas l'esprit: il n'y a pas de vertu aux privations. » C'est contraire à ce qu'on entend dire habituellement, notamment aux paroles de nombreux grands mystiques.

Amélie Nothomb: Absolument. C'est du moins ce que j'ai découvert durant cette longue expérience, puisque de treize à quinze ans je n'ai pas mangé. Depuis, je suis très irritée lorsque j'entends des éloges d'ascèse, voire des éloges de l'anorexie qui sont de plus en plus nombreux. Au cours de l'anorexie, la seule chose que j'ai découverte est qu'il n'y a aucune vertu à l'anorexie.

Josyane Savigneau : Avez-vous jugé de cela a posteriori? Ne donniezvous aucune vertu à cette ascèse au moment où vous le viviez? N'aviezvous pas l'impression que c'était quelque chose de magnifique à ce moment-là ?

Amélie Nothomb: Non, je m'en suis rendu compte tout de suite car même s'il y a des moments d'exaltation dans l'anorexie, ces moments ne peuvent pas 
voiler combien le corps et l'esprit sont diminués. On s'imagine que l'on va trouver des trésors d'esprit dans l'ascèse. C'est le contraire en réalité. On se trouve réduit à sa plus simple expression. Lâme se trouve réduite à sa portion congrue. Peut-être qu'il existe de très grands mystiques pour lesquels cela ne s'est pas passé ainsi, ou peut-être que tout le monde n'est pas fait pour devenir de grands mystiques.

Josyane Savigneau: Vous décrivez la difficulté physique de recommencer à manger. Vous souvenez-vous du moment où vous l'avez décidé?

Amélie Nothomb: Je m'en souviens parfaitement comme du fait que je ne l'ai pas décidé. Je l'ai vécu de façon schizophrénique puisque mon corps et mon âme se sont séparés. Mon corps, motivé par l'instinct de survie, est allé manger car c'est tout ce qu'il a trouvé pour ne pas mourir. Je ne souhaite à personne une telle expérience. La lente reconquête du corps et de la nourriture et d'un semblant de rapport entre le corps et l'âme sont très difficiles.

Josyane Savigneau : Au moment où vous lisez La Chartreuse de Parme, vous dites: «Il n’y a pas plus civilisé que ce livre. L'anorexie me tenait à l'écart de la civilisation et j'en souffrais. "

Amélie Nothomb : Effectivement, il y a aussi toute cette dimension antisociale de l'anorexie. Dès l'instant où vous cessez de manger, les 11 gens ne veulent plus vous parler. Vous ne faites plus partie de leur Amélie Nothomb groupe et je n'ai jamais été le genre de personne qui ne veut pas faire Entretien avec partie du groupe. Être complètement mis à l'écart est une souffrance Josyane Savigneau supplémentaire.

Josyane Savigneau: Avez-vous senti une exclusion par rapport à vos parents?

Amélie Nothomb : Mes parents ne s'en rendaient pas vraiment compte sinon ils m'auraient mise dans une clinique.

Josyane Savigneau : Votre sœur a-t-elle fait la même chose que vous et aussi longtemps?

Amélie Nothomb: Pour ma sœur, c'est différent: comme elle n’a jamais fait cela de façon aussi « jusqu'au-boutiste » que moi, elle ne s'en est jamais véritablement sortie.

Josyane Savigneau : Vous imposiez-vous un rationnement?

Amélie Nothomb : Absolument. J'avalais à peine de l'eau. Vous n'avez pas idée jusqu'où allaient ces privations. C'étaient des mortifications qui ont duré très longtemps. 
Josyane Savigneau: Vous parlez des Jeunes Filles de Montherlant, lecture d'adolescente. N'est-ce pas un livre terrible pour les femmes?

Amélie Nothomb: Je l'ai lu plus de cent fois et je continue à le relire régulièrement. Cependant, je ne l'ai jamais lu autant et si compulsivement qu'à l'adolescence. Je trouve normal, surtout à cet âge, d'être obsédé par ce livre qui fait un diagnostic épouvantable de la condition féminine de cette époque, même si je ne suis pas sûre que l'on en soit totalement sorti.

Josyane Savigneau : Ce n'est pas qu'un diagnostic de la condition féminine. C'est un diagnostic de la psychologie féminine, qui pour Montherlant pouvait traverser les siècles. Il avait une détestation pour la psychologie féminine que l'on peut ne pas partager, mais il est vrai qu'il est plutôt séduisant à l'adolescence. Très souvent, on lit des romans à charge contre les femmes. On les juge outrageusement misogynes, puis on voit des stéréotypes de ces femmes dans la réalité. Je crois que la détestation que provoque Simone de Beauvoir chez un certain nombre de "supposés féministes " aujourd'hui est qu'elle n’a pas dit, dans Le Deuxième Sexe: «Femme, parle, la vérité sort de ta bouche " mais: "Femmes, vous êtes aliénées, il faudrait en sortir". Des lectures comme celles de Montherlant ont-elles suscité des personnages comme celui d'Antéchrista?

Amélie Nothomb: Non, j'ai connu le personnage d'Antéchrista. J'ai vécu l'histoire de Blanche. Je dirais même que c'est une histoire vécue surtout une fois, mais à ma manière je l'ai vécue un grand nombre de fois également.

\section{2}

Amélie Nothomb

Entretien avec

Josyane Savigneau : Antéchrista est-elle l'emblème de plusieurs personnages Josyane Savigneau réunis? Est-elle ce que l'on peut détester chez une femme?

Amélie Nothomb: Oui, elle est l'emblème. Cependant, elle n'est pas totalement détestable. Elle est très séduisante par certains aspects; bien qu'elle arrive, prenne le pouvoir et vous mette dehors. Je connais cela.

Josyane Savigneau : Elle a également une idée vaniteuse de sa propre représentation, de son image. N'est-ce pas cela qui est odieux?

Amélie Nothomb : En soi, cela pourrait être mignon si ça ne s'exerçait pas de façon tellement méprisante envers l'autre. D'autant plus que l'héroïne est une adolescente. Ce n'est pas seulement "Je suis sublime ", mais " Je le suis car tu es nulle ». Cette situation est donc très difficile à vivre.

Josyane Savigneau : Sur le thème du corps, Le Robert des noms propres est assez terrible également.

Amélie Nothomb: Oui. Il raconte l'histoire d'une grande amie, la chanteuse Robert, qui avant de devenir cette chanteuse, était petit rat de l'Opéra. Elle m’a raconté son histoire, ce qui se passait dans les années 1970 à l'École de danse de l'Opéra de Paris où il y avait vraiment une haine du corps contraire, 
je crois, aux Droits de l'homme. C'est d'autant plus effrayant de voir une mère approuver les sévices faits à sa fille et la détester si celle-ci veut retrouver un corps normal. Ce personnage est effrayant.

Josyane Savigneau: Ce qui vous intéressait dans ce livre était le travail sur le corps, mais n'était-ce pas également les rapports terribles que peuvent entretenir mères et filles?

Amélie Nothomb: L'amour qui peut exister entre une mère et sa fille est l'une des formes les plus paroxystiques de l'amour. C'est rarement simple et souvent passé sous silence.

Josyane Savigneau : N'y a-t-il pas chez cette mère une volonté que sa fille fasse ce qu'elle a toujours rêvé de faire, à n'importe quel prix ?

Amélie Nothomb: Oui. Il est magnifique de rêver d'avoir un enfant. En revanche, il est dangereux de rêver un enfant. Personnellement, je n'ai pas d'enfants et je n'en aurai pas mais je pense que c'est une chose qui arrive souvent. Cependant, devenir mère est certainement très difficile.

Josyane Savigneau : La manière d'allier beauté et laideur, de voir la laideur dans la beauté, de chercher la beauté dans la laideur est courant chez vous également. Pouvez-vous nous parler de Mercure et Attentat?

Amélie Nothomb: Le premier des deux, Attentat, narre l'homme le plus 13 laid du monde qui tombe amoureux de la plus belle femme du monde. Amélie Nothomb Dans le livre suivant, Mercure, encore plus poussé à l'extrême, on obtient Entretien avec une synonymie entre le comble de la laideur et celui de la beauté. Suite à des Josyane Savigneau manipulations narratives, on se rend compte que la femme du livre, censée être d'une laideur sans nom, est en fait la plus belle femme du monde.

Josyane Savigneau : N'est-ce pas dans Mercure que vous parlez de « la beauté au cœur de l'immonde »?

Amélie Nothomb: Oui, il y a beaucoup de façons d'explorer cela dans ma vie: le Japon et la Belgique, par exemple. Le Japon, plus qu’aucun autre pays, a élevé un piédestal à la beauté. Pourtant, la laideur y est présente. Il est donc très singulier de se promener dans ce pays magnifique et d'y voir la laideur. La Belgique est presque le contraire: un pays où la laideur circule mais où tout à coup on voit la beauté. Il réside un contraste entre les deux.

Josyane Savigneau: Vous avez vécu dans beaucoup de pays, pourtant le Japon et la Belgique sont les deux pôles? Pourquoi?

Amélie Nothomb: Mon père était ambassadeur de Belgique, il représentait ce pays. Il en parlait beaucoup. Je savais, sans le comprendre, que je venais de ce pays. Désormais, je suis convaincue que cette identité me va très bien même si je ne comprends toujours pas vraiment ce que veux dire "être belge ». Mais cette bipolarité complètement absurde me résume bien. Vous 
chercheriez un lien philosophique, historique, esthétique entre le Japon et la Belgique, vous n'en trouveriez pas. Il est absurde d'être à la fois belge et japonais. Ce sont des pays qui n'ont pas de rapports.

Josyane Savigneau : Le Japon est le lieu de votre enfance. Ensuite, il y a eu tous ces pays, notamment les États-Unis. Vous dites avoir aimé New York.

Amélie Nothomb: Oui, mais il n'y a aucun pays dans lequel l'identification a été faite comme au Japon. J'imagine que je fais partie de ces espèces fossiles qui sont surtout attachées à leur toute petite enfance. Mais c'est aussi que ce pays est le Japon: pays capable d'exercer un pouvoir de fascination à nul autre pareil.

Josyane Savigneau : Vous n'avez donc pas eu, dans les autres pays, ce sentiment d'appartenance?

Amélie Nothomb : C'est cela. Au Japon, j’y étais née, mes parents m’avaient inscrite à l'école japonaise alors qu'ils avaient mis mon frère et ma sœur à l'école américaine. J'avais donc le sentiment d'une élection. Il y a un rapport profond entre ce pays et moi, du moins c'est ce que je pensais à l'époque. En plus, ma gouvernante japonaise m'aimait comme si j'étais sa fille et je l'aimais comme ma mère. Mes parents voyaient en moi une vocation japonaise du fait d'être née au Japon.

Josyane Savigneau : Avant de découvrir le sentiment de « belgitude », vous vous êtes sentie japonaise.

\section{4}

Amélie Nothomb

Entretien avec

Amélie Nothomb: Oui, avec beaucoup d'obstination et plus longtemps Josyane Savigneau que l'on y a cru. Jusqu'à mon retour du Japon, à l'âge de vingt et un ans, j'ai entretenu très intimement cette mythologie d'être japonaise. Je ne le disais pas car, au fil des années, je voyais la perplexité dans le regard des autres lorsque je disais que j'étais japonaise. Cependant, je le pensais d'autant plus au fond de moi.

Josyane Savigneau: Malgré tout, vous sentez-vous toujours partiellement japonaise?

Amélie Nothomb : Oui. Il y a toujours beaucoup de Japon en moi et il est le pays que j'aime le plus au monde. Mais j'ai appris à vivre cet amour de loin.

Josyane Savigneau : Qu'en est-il pour la Belgique?

Amélie Nothomb : J'ai fini par comprendre, ne serait-ce que par élimination, que c'était mon pays. Je le crois d'autant plus à l'heure où ce pays est en crise. Le fait que cette identité belge soit si problématique me permet de mieux m’identifier à ce pays.

Josyane Savigneau : Parlez-vous les deux langues de la Belgique?

(C) Éditions de la Bibliothèque publique d'information/Centre Pompidou, 2010

ISBN 978-2-84246-131-7 
Amélie Nothomb: Malheureusement non. Je suis arrivée trop tard en Belgique pour apprendre le néerlandais, mais je le comprends un peu. Je le regrette beaucoup d'autant plus que ma famille maternelle est flamande.

Josyane Savigneau : Avez-vous ce sentiment d'appartenance physique à la Flandre, aux paysages?

Amélie Nothomb: Je constate seulement une chose: aucune peinture au monde ne provoque une réaction aussi forte en moi que la peinture flamande.

Josyane Savigneau: Marguerite Yourcenar, par exemple, avait très peu vécu en Belgique et n'était pas belge de nationalité puisque son père était français. Malgré tout, chaque fois qu'elle y revenait, elle avait ce sentiment étrange et cet attachement à la peinture flamande que vous avez. Comment l'expliquez-vous?

Amélie Nothomb: Il doit y avoir quelque chose. Ce n'est pas normal qu'en n'ayant jamais été formée à cette peinture, et n'étant pas vraiment sensible aux arts plastiques, elle me fasse tellement d'effet.

Josyane Savigneau: Cosmétique de l'ennemi n'est-il pas un livre énigmatique?

Amélie Nothomb: Il est venu d'une grande colère. Je compare chacun de mes livres à des grossesses et ce qui m’a mise enceinte de ce livre est un état de haine implacable. Il commence avec le thème de l'intrus, de l'importun, Amélie Nothomb typique dans mes livres. Un type attend son avion à l'aéroport. L'avion est Josyane Savigneau annoncé en retard. Il prend son mal en patience. Un autre homme arrive et entame la conversation contre son gré - situation que j'ai vécu très souvent dans ma propre vie. Au début, le premier homme essaie de se débarrasser de l'autre puis il comprend qu'il n'y parviendra pas. Il fait donc contre mauvaise fortune bon cœur et subira l'intrus comme il le pourra. Jusqu'au moment où l'intrus se met à dire des choses étranges qui tendraient à prouver qu'il a une connaissance intime de sa vie à lui et la situation dégénère.

Josyane Savigneau: En fait, c'est le même homme et il devra mourir. D'autre part, Stupeur et Tremblements et Ni d'Ève ni d'Adam sont différents mais peuvent être rapprochés. Vous avez écrit sur la quatrième de couverture de Ni d'Eve ni d'Adam: "Stupeur et Tremblements pourrait donner l'impression qu'au Japon, à l'âge adulte, j'ai seulement été la plus désastreuse des employés. Ni d'Eve ni d'Adam révélera qu'à la même époque et dans le même lieu, j’ai été aussi la fiancée d'un Tokyoïte très singulier. » Peut-on dire que ces livres fonctionnent comme une sorte de diptyque? Stupeur et Tremblements n'est-il pas une sorte de polar psychologique?

Amélie Nothomb: Un polar sur le harcèlement moral dans une entreprise. 
Lecture du début de Stupeur et Tremblements par Amélie Nothomb:

Monsieur Haneda était le supérieur de Monsieur Omochi, qui était le supérieur de Monsieur Saito, qui était le supérieur de Mademoiselle Mori, qui était ma supérieure. Et moi, je n’étais la supérieure de personne...

(Écouter la suite sur archives sonores, repère: 47 min 47 s)

Josyane Savigneau: Ce n'est pas seulement le monde de l'entreprise en général. N'y a-t-il pas également la particularité qu'ont les Japonais dans la manière d'aborder le travail et les rapports hiérarchiques?

Amélie Nothomb: Le respect de la hiérarchie compte infiniment plus dans l'entreprise japonaise que la poursuite de l'efficacité ou du profit. On peut vraiment considérer l'entreprise japonaise comme une tentative de structure de la société avec parfois, accidentellement, du profit.

Josyane Savigneau : Pourtant, l'argent n'apparait-il pas comme un personnage important dès le début? "L Largent, chez Yumimoto, dépassait l'entendement humain ", dites-vous. "À partir d'une certaine accumulation de zéros, les montants quittaient le domaine des nombres pour entrer dans celui de l'art abstrait.

Amélie Nothomb: C'était encore l'époque de la «Bubble economy ", la crise n'avait donc pas encore commencé au Japon. Les sommes d'argent manipulées étaient énormes. Cependant, dans la même situation, des Chinois auraient manipulé des sommes d'argent beaucoup plus importantes. L'argent n'était vraiment pas une obsession.

\section{6}

Amélie Nothomb

Entretien avec

Josyane Savigneau

Josyane Savigneau: N'est-ce pas un roman de critique sociale sur le monde de l'entreprise?

Amélie Nothomb: Oui, ce serait un tort de le limiter à la sphère japonaise. Cette entreprise était exemplaire à cette époque, dans le sens où jamais on ne licenciait. Les plans sociaux sont très récents au Japon. Jusqu'il y a peu, on considérait que l'on avait le droit d'aller très loin avec les employés mais pas de les licencier. C'était considéré comme beaucoup trop inhumain.

Josyane Savigneau: Oui, enfin il peut être inhumain de garder les gens par moments. Par exemple, le reproche est fait à l'héroïne, vous, d'être individualiste.

Amélie Nothomb: Oui, mais il est vrai que les Japonais ne le sont vraiment pas. Leur système s'explique ainsi. Le mot "individualiste " est vu comme une insulte, un défaut. J'ai identifié que je n'étais pas japonaise en découvrant que, malgré tout, je suis individualiste.

Josyane Savigneau : Tout un réseau de dénonciation est présent également dans cette entreprise. 
Amélie Nothomb: Effectivement, j’ai été étonnée de voir que le code d'honneur japonais admettait la délation, emblématique du harcèlement moral.

Josyane Savigneau : Puis, vous avez été sanctionnée pour erreur de comptabilité, poste que l'on vous a attribué par dépit puisque vous pensiez être employée comme traductrice. Que s'est-il passé?

Amélie Nothomb: Oui, on a vu mon ignorance et ma bêtise en matière de comptabilité - confondre l'entreprise allemande GMH avec l'appellation Société Anonyme - comme un acte de sabotage. Tout le monde m'a pris pour une saboteuse et pour me punir j'ai été rétrogradée en "dame pipi ", et cela pendant sept mois de ma vie. Me mettre d'abord à la comptabilité puis aux toilettes était une drôle d'idée.

Josyane Savigneau : Au fond, vous dites quelque chose de dur sur les lieux autoritaires.

\footnotetext{
Je rentrai dans le siècle. Il peut paraitre étrange que, après ma nuit de folie, les choses aient repris comme si rien de grave n'était arrivé. Certes, personne ne m'avait vu parcourir les bureaux toute nue, en marchant sur les mains, ni rouler un patin à un honnête ordinateur...

(Écouter la suite sur archives sonores, repère: $54 \mathrm{~min} 50 \mathrm{~s}$ )
}

Amélie Nothomb : Le Japon multiplie ces exemples. Il y a le cas célèbre $\mathrm{du}$ Japonais anthropophage, qui avait mangé sa petite amie hollandaise en racontant comment il l'avait découpée et avait cuisiné chaque morceau. Amélie Nothomb Quand il a été capturé par la police, il est passé en jugement et il s'est Josyane Savigneau expliqué de son acte devant les autorités japonaises. Il a reçu une peine minime et, une fois sa peine purgée, il fut embauché comme chroniqueur gastronomique. Je n'étais donc pas le plus grand cas de déviance du système japonais.

Josyane Savigneau : Avez-vous tout de même perçu une autorité stricte au Japon?

Amélie Nothomb: Indubitablement, l'autorité ne se discute pas. Dans Stupeur et Tremblements, on me voit souvent dans cette situation d'erreur flagrante de tenter de raisonner l'autorité, de la discuter. À chaque fois, en contrepartie, j'ai eu des châtiments sans bornes car c'était la preuve que je remettais en cause la suprême hiérarchie. L'application est la même à l'école: on ne discute pas avec le professeur japonais si on ne comprend pas ce qu'il explique. Prendre la parole en classe serait vu comme la remise en cause de son autorité.

Josyane Savigneau : Cette manière de vouloir discuter l'autorité était perçue, dites-vous, comme l'expression de l'infériorité du cerveau occidental. Pour autant, à votre départ on ne vous a pas fait de reproches. Peut-on parler d'hypocrisie? 
Amélie Nothomb : Non, je me suis même aperçue que l'on m'aimait plutôt bien. Ils avaient l'air de me trouver divertissante. Ils m'auraient même certainement gardée si je n'avais pas décidé de partir. Il y a tout un processus pour quitter l'entreprise: donner maintes fois sa démission en suivant la hiérarchie (supérieur direct puis le supérieur du supérieur, etc.), s'accabler d'être assez fou pour vouloir quitter ce magnifique tissu social. J'étais excellente à ce jeu. Je démissionnais du poste de "dame pipi ». Dire que l'on ne dispose pas des capacités intellectuelles requises pour un tel poste était grisant.

Josyane Savigneau : Écriviez-vous déjà à cette période de votre vie?

Amélie Nothomb : J'écrivais déjà depuis plusieurs années. J'avais déjà écrit quelques-uns des livres, abandonnés dans les cartons, mais aucun de ceux qui ont été publiés.

Josyane Savigneau : À plusieurs moments, vous avez comparé la manière de créer à une grossesse. Peut-on dire que vous avez abandonné beaucoup d'enfants?

Amélie Nothomb : Non, car je ne considère pas cela comme des abandons. Ce sont des " enfants" menés à terme. Je ne suis pas sûre que ce serait un acte de "bonne mère " de considérer que tous ses enfants sont destinés à la lumière, à la publication. Certains sont faits pour l'ombre. Je les aime tous autant.

Josyane Savigneau : Ni d'Ève ni d'Adam, votre dernier livre autobiographique, Entretien avec constitue-t-il le versant enchanté de cette expérience japonaise? N'est-il pas Josyane Savigneau également mélancolique parfois?

Amélie Nothomb : Lorsque j'ai écrit les dernières pages de ce livre, je sanglotais. Ce fut difficile d'écrire entre ces larmes. Il est vrai que ce garçon [Rinri] était charmant. Tous ceux qui ont lu ce livre se sont demandés pourquoi je l'avais quitté, ce charmant garçon. Je pense que le fait d'être partie rend l'histoire si parfaite.

Josyane Savigneau : C'est un livre dont on a l'impression, à chaque phrase, que vous avez pris plaisir à l'écrire, à raconter cette histoire. Qu'en pensezvous?

Amélie Nothomb: J'ai adoré vivre cette histoire merveilleuse. J'ai adoré lui redonner vie par l'écriture. Il y a cette phrase de Virginia Woolf: "Il ne s'est rien passé aussi longtemps qu'on ne l'a pas écrit. " J'ai pris une intense jouissance à l'écrire seize ans après les faits.

Lectures de Ni d'Ève ni d'Adam par Josyane Savigneau:

Je courus chercher du Coca au frigo, assurant que cela allait très bien avec la fondue suisse, et remplis mon verre à pied...

(Écouter la suite sur archives sonores, repère: 1 h 02 min 20 s)

(C) Éditions de la Bibliothèque publique d'information/Centre Pompidou, 2010

ISBN 978-2-84246-131-7 
L'amour est un élan si français que d'aucuns y ont vu une invention nationale. Sans aller jusque-là, je reconnais qu'il y a dans cette langue un génie amoureux...

(Écouter la suite sur archives sonores, repère: 1 h 03 min 25 s)

Amélie Nothomb: J'ai essayé la pratique de la déclaration d'amour dans plusieurs langues. On sent une tradition, une éloquence amoureuse française. C'est même plus fort que la langue car les Belges sont moins bien. Rinri a su apprendre cette langue grâce à mes leçons. Je l'ai retrouvé plus tard. Cette scène était bouleversante. En l'écrivant, j'ai pleuré toutes les larmes de mon corps.

Josyane Savigneau : Pourquoi dites-vous dans ce livre: «Il n'est pas banal que j'écrive une histoire où personne n'ait envie de massacrer personne "?

Amélie Nothomb : Rassurez-vous, dans ce que j'écris actuellement tout le monde veut se massacrer.

Josyane Savigneau : N'avez-vous plus de livres enchantés comme Ni d'Ève ni d'Adam?

Amélie Nothomb : Momentanément, non. Cependant, sait-on jamais de quoi l'on tombera enceinte? Mes cycles ne sont jamais finis. J'ai des cycles infernaux, démoniaques qui seront toujours féconds et, à l'instar, des cycles tout à fait charmants. Il y a tout ça en moi.

Josyane Savigneau: Je voudrais qu'on termine sur un livre très méchant, Entretien avec diversement apprécié, fort dans la critique sociale: Acide sulfurique. Vous avez Josyane Savigneau poussé la critique sociale à son paroxysme. Vous êtes partie de l'idée de la télé-réalité, où des gens acceptent d'être humiliés pour passer à la télévision. Vous avez considéré que son extrême était le camp de concentration.

Amélie Nothomb: J'ai pris le point de vue "jusqu'au-boutiste » de la chose. Dans mon livre, l'émission de télé-réalité met en scène un camp de concentration. La seule différence est que les gens ne sont pas volontaires. La télévision procède à de véritables rafles pour les mettre dans ces camps. Les gens sont filmés continuellement, première souffrance, et ils ne sont jamais autant filmés que quand ils souffrent. Tous les jours des gens sont tués et, au moment où les gens de la télévision sentent que l'audience baisse, ils comprennent que pour stopper le phénomène il faut que ce soit les téléspectateurs qui choisissent les condamnés.

Josyane Savigneau : Vous dites: « La souffrance des autres ne leur suffit plus, il leur fallut le spectacle. » Dénoncez-vous la mise en spectacle de situations tragiques par la télé-réalité?

Amélie Nothomb : Je trouve hallucinant que certaines télévisions essaient de faire croire qu'il y a un autre but à ce genre d'émissions que le sadisme pur et simple. Si au moins il y avait un aveu de sadisme cela passerait peut-être un 
peu mieux. La plupart du temps, ils veulent nous faire croire que ces émissions ont des intentions bienfaisantes: apprendre aux gens à s'habiller alors que de toute évidence ils ne cherchent qu’à les humilier et montrer comme ils sont laids. Il est singulier qu'en démocratie il y ait des volontaires pour ce genre d'expérience. Cela m'a intriguée au point d'y consacrer un livre. Livre qui n'est pas si « jusqu'au-boutiste » que cela puisque ce qui permet à n'importe qui d'aller jusqu'au bout de la barbarie est d'accepter le premier échelon. Il me semble que le premier échelon, nous l'avons tous franchi depuis longtemps. Je ne vois donc pas l'aberration de ce livre.

Josyane Savigneau : Avez-vous déjà regardé des émissions de télé-réalité?

Amélie Nothomb : Il n'était pas nécessaire d'en avoir regardé. Je ne sais pas si ce phénomène s'est calmé, du moins je l'espère. Au début des années 2000, je constatais que les gens ne parlaient que de cela. Je savais tout ce qui s'était passé dans ces émissions rien qu'en écoutant les conversations des gens.

Josyane Savigneau : Dans le livre, il y a une petite différence par rapport à la télé-réalité : les gens sont volontaires habituellement.

Amélie Nothomb : Comment ne pas s'interroger sur la notion de volontarisme lorsqu'on voit ce que ces gens consentent à faire? Il y a de quoi s'interroger sur la définition de liberté humaine.

Josyane Savigneau: Peut-on rapprocher vos propos de la fameuse phrase 2 d'Andy Wharol: "À l'avenir, chacun aura son quart d'heure de célébrité mondiale »?

Amélie Nothomb : Oui. Seulement, autant cette phrase peut être vue comme sympathique et anecdotique, autant ses dérives modernes sont effrayantes.

Josyane Savigneau : Avez-vous eu des réactions de lecteurs choqués?

Amélie Nothomb : Bien sûr. De façon symptomatique, les gens qui ont protesté le plus fort et avec le plus d'indignation contre ce livre étaient des personnes qui travaillaient dans les médias, surtout la télévision.

Josyane Savigneau : La critique sociale est souvent présente dans vos livres, malgré l'humour et l'ironie. N'avez-vous pas été un peu loin avec ce livre?

Amélie Nothomb : C'est un thème très grave, qui dépasse de très loin la télévision. Il s'agit du thème du mépris, organisé par des pans entiers du divertissement. Il y a de quoi en avoir froid dans le dos. Accepter de se laisser filmer pour être humilié est très troublant. 
Public : Comment vos personnages se développent-ils quand vous sentez que l'histoire arrive à son climax ? Comment arrivez-vous à trouver une fin?

Amélie Nothomb : Je réfléchis très peu à ce genre d'interrogations. Ce n'est pas pour rien que j'emploie toujours la métaphore de la grossesse. Il y a un travail qui m'accapare profondément pendant un certain nombre de mois. Même quand je n'écris pas, l'essentiel de mon être écrit sans que je m'en rende compte. C'est devenu une espèce de processus organique qui réquisitionne mon esprit inconsciemment. Je ne vais pas dire que je n'exerce aucun contrôle conscient de la situation mais j'ai le sentiment que la réflexion y apporte très peu. $\mathrm{Ma}$ réflexion n'est pas consciente. Je sais que j'arrive à terme au moment où je vois le bébé en son entier, comme dans le cas d'un accouchement. Je n'ai jamais eu ce questionnement, qu'ont de nombreux écrivains, sur «Comment savoir quand son livre est fini ? " Je n'ai jamais eu d'hésitations. Je sais que mon livre est fini.

Public : De quel livre êtes-vous la plus fière?

Amélie Nothomb : Très sincèrement, il n'existe pas de livre pour lequel je sois le plus ou le moins fière. Je pense, en cela, être une "bonne mère ". Je suis capable de reconnaître que j'ai des livres plus beaux, plus charmants, plus intelligents que d'autres. Cependant, je ne suis pas capable de les préférer. De même, je crois qu'une mère ne préfère pas ses plus beaux enfants ou les plus intelligents. J'ai des rapports très forts et évolutifs avec tous mes livres, y compris ceux que je ne publie pas. Je n'ai jamais de rapports stables vis-à-vis de mes livres. De toute façon, tous mes livres sont passés par une phase de détestation, voire de haine.

Josyane Savigneau : Lisez-vous vos livres à haute voix ?

Amélie Nothomb : Non, quelle horreur! Je n'ai fait cela ce soir que pour vous complaire. J'entends la phrase quand je l'écris dans cette espèce de silence intérieur. J'ai horreur de ma voix. Si je devais entendre mes phrases avec ma voix, je pense que cela me bloquerait complètement. Je les entends, néanmoins, intérieurement avec une autre voix, qui est beaucoup plus la mienne.

Public: Vous dites que "la métaphore est de la mauvaise foi ", alors pourquoi l'utilisez-vous autant? Est-ce de l'ironie?

Amélie Nothomb : Je pousse la 21

Amélie Nothomb mauvaise foi à son comble jusqu'à Entretien avec utiliser des métaphores moi-même. Josyane Savigneau Ce n'est pas ironique. En revanche, dans Hygiène de l'assassin, j'ai une réaction contre l'abus de la métaphore. J'ai fait des études dans lesquelles certains professeurs de lettres abusaient de la métaphore. Je ne parle pas seulement dans leur expression mais surtout dans leurs explications. Bien sûr, la métaphore est essentielle à la littérature et il n'y a pas de littérature sans métaphores. D'autre part, recourir seulement à la métaphore dans l'explication de texte est insoutenable, et la mort du sens. Je réagissais donc contre cet excès. Évidemment, Prétextat est "jusqu'au-boutiste" dans sa façon de vivre. Il ne faut pas aller aussi loin que lui. Regardez, par exemple, sa façon de se nourrir ; il est le même quand il parle. 
Public: Avez-vous fluidifié votre écriture, dans le sens où vos derniers livres comprennent moins de mots savants que les premiers? Est-ce une volonté de toucher un public plus large?

Amélie Nothomb : Écrire dans le but d'avoir un public plus large est bien la chose qui me viendrait en dernier à l'esprit. C'est mal me connaître. Si tel est le cas, c'est une simple coïncidence car je n'ai jamais eu de telles intentions. Je pense que mon écriture évolue. Ce serait dommage de tant écrire sans évoluer. Mais permettez-moi de prendre négativement votre remarque. L'écrivain qui cible quelque chose est sûr de le rater, et heureusement. Il n'y a que les «faiseurs de livres" qui pensent à leur public.

Public: Pourriez-vous dire un mo du violoncelle à la fin d'Acide sulfurique?

Amélie Nothomb : Il fallait que ce livre se termine de façon profondément humaine. J'en suis arrivée à la conclusion qu'il n'y avait rien de plus humain au monde qu'un violoncelle. C'est une création de l'homme. Puis l'homme n'est jamais aussi humain que lorsque l'on entend sa voix. Or, de tous les instruments, le violoncelle est celui qui s'approche le plus de la voix humaine. J'ai voulu que ce livre se termine ainsi car la voix humaine est ce qui fait le plus de bien.

Public : Quel accueil a reçu au Japon Stupeur et Tremblements?

Amélie Nothomb : Ce qui est déjà extraordinaire, c'est que le livre y ait été reçu. Si j'avais consacré un tel livre à la Chine, elle ne l'aurait pas reçu. Les Japonais prouvent ainsi qu'ils ont une belle ouverture d'esprit. Ils s'intéressent à ce que l'on pense d'eux, au point d'acheter les droits du livre et de le traduire. Je considère donc que mon but est atteint. Par ailleurs, soyons réalistes, ce livre n'a pas été aimé des Japonais, à quelques rares exceptions près. Cependant, ce n'était pas le but. Cela a donné lieu à un débat houleux au Japon et, depuis lors, je refuse toutes les interviews japonaises.

Public : Avez-vous rencontré Haruki Murakami ?

Amélie Nothomb : Malheureusement, non. Haruki Murakami est une immense star de la littérature mondiale. Il est tout à fait impossible pour une humble Belge comme moi de dialoguer avec lui mais je ne désespère pas un jour d'y parvenir. J'en serais très heureuse. Cependant, j'ai rencontré plusieurs fois Kenzaburô Ôé mais n'ai jamais eu véritablement d'entretien littéraire avec lui.

\section{Amélie Nothomb}

Entretien avec

Public: Vos grossesses de livres fictionnels sont-elles différentes de vos grossesses de livres autobiographiques?

Amélie Nothomb : Effectivement, il m'a toujours fallu plus de temps pour écrire des autobiographies que de la fiction. Dans le cas de la fiction, le problème de la frontière ne se pose pas : on peut vraiment tout dire. Dans le cadre d'une écriture autobiographique, je pense qu'il y a ce problème qui intervient, même si ce n'est pas le cas pour tous les écrivains. Certains pensent qu'il ne faut pas tenir compte de cette frontière. C'est donc tout un art, proprement géographique, d'apprendre à dessiner la juste frontière. 
Public : Je suis japonais et je voudrais savoir ce que vous pensez des Japonaises. Pensez-vous que la majorité des femmes japonaises sont contre l'antiféminisme de la société japonaise?

Amélie Nothomb : Je ne peux prétendre connaître les Japonais mieux que vous mais au Japon tout est représenté à son comble. Bien sûr qu'il existe des carcans dont souffrent les femmes japonaises, décrits dans Stupeur et Tremblements. À côté de cela, des Japonaises féministes sont présentes et vont très loin dans leur féminisme. Il existe des courants féministes comme l'école de Takarazuka ${ }^{4}$, qui existe depuis longtemps.

Public : Quel est votre rapport entre le pacte autobiographique auteur/ lecteur et la volonté graphomane d'écrire, qui est à la fois cathartique et extatique?

Amélie Nothomb : Mon pacte autobiographique est instinctif mais je ne sais pas s'il est didactique. Tout ce que je dis dans mes livres est vrai mais je ne dis pas toute la vérité. Je veux garder des choses pour moi. C'est le seul pacte que j'ai pu élucider dans mon écriture autobiographique.

Josyane Savigneau : Écrivezvous avec du chocolat dans l'autre main?

Amélie Nothomb : Écrire et manger en même temps est impossible. Pour écrire, j'ai besoin d'avoir très faim. J'entretiens cette faim en ne mangeant pas aussi longtemps que je n'ai pas fini d'écrire.

Public : Pouvez-vous nous parler de l'ouvrage Les Combustibles?
Amélie Nothomb : Les Combustibles est le seul texte que j'ai écrit a priori pour le théâtre. Je l'ai écrit pendant le siège de Sarajevo ${ }^{5}$, qui m'a inspiré. J'avais lu dans un journal : " Sarajevo, les gens ont si froid qu'ils brûlent leurs livres. " Cette phrase m'est restée en travers de la gorge et pour la comprendre j'ai écrit cette pièce de théâtre, mettant en scène trois individus enfermés dans un appartement l'hiver. Ils n'ont plus d'autre combustible que leurs livres et discutent avec le peu d'humanité qui leur reste pour savoir quels livres ils vont sacrifier. Peut-être écrirai-je encore du théâtre? Comme je le disais, on ne sait jamais de quoi l'on tombera enceinte.

Public : Dans vos œuvres, les livres ont une grande importance : il y a les livres qui sauvent dans Les Combustibles et les livres qui tuent dans Journal d'hirondelle. Quels sont 23 ceux qui vous ont sauvée?

\section{3}

Amélie Nothomb Entretien avec

Amélie Nothomb : Une foule de Josyane Savigneau livres m'ont sauvée, surtout ceux lus pendant mon adolescence: Stendhal, Montherlant, Woolf... Mon adolescence, interminable et ô combien solitaire, a prouvé qu'on pouvait être sauvé tous les jours par des livres. On ne peut pas vivre dans le néant, surtout pas à l'adolescence. Ma reconnaissance est infinie envers tous ces immenses auteurs qui m'ont tirée du néant et permis de l'affronter. Cependant, j'ai envie d'avoir de la reconnaissance pour tous les livres, même les mauvais. Il y a une jouissance du mauvais livre, un apprentissage. Ils nous apprennent ce qu'il ne faut pas écrire. 


\section{Notes}

1 Lacan (1901-1981) refusa souvent d'être publié pour rester cohérent avec l'enseignement de la psychanalyse fondé sur le colloque et l'émergence de la vérité au détour du surgissement de la parole. Il qualifiait ainsi la littérature psychanalytique de "poubellication ». $\uparrow$

${ }^{2}$ Michel David, Amélie Nothomb: le symptôme graphomane, L'Harmattan (L'Cuvre et la Psyche), 2006. [livre en ligne] $\uparrow$

${ }^{3}$ Sorte de bulle spéculative où le commerce des produits et des biens se fait avec des valeurs gonflées. $\uparrow$

${ }^{4}$ À l'origine, troupe de théâtre qui coïncide avec l'émergence des Japonaises dans le monde du travail et l'apparition des « modern-girls » revendiquant une liberté sexuelle défiant le modèle de la « bonne épouse et mère avisée ». La revue est composée d'actrices uniquement, qui doivent toutes être célibataires. Elles apprennent à se comporter en hommes et jouent sur la frontière entre les identités sexuelles pour faire, de la manipulation des genres, l'essence du spectacle. $\uparrow$

${ }^{5}$ Du 5 avril 1992 au 29 février 1996, un blocus fut établi par les forces serbes contre la Bosnie-

Herzégovine qui venait d'obtenir son indépendance par rapport à la Yougoslavie. $\uparrow$ 